\title{
Automatically selecting the best pictures for an individualized child photo album
}

\author{
Floris De Feyter, Kristof Van Beeck, and Toon Goedemé \\ KU Leuven - EAVISE, Belgium, \\ floris.defeyter@kuleuven. be
}

\begin{abstract}
In this paper we investigate the best way to automatically compose a photo album for an individual child from a large collection of photographs taken during a school year. For this, we efficiently combine state-of-the-art identification algorithms to select relevant photos, with an aesthetics estimation algorithm to only keep the best images. For the identification task, we achieved $86 \%$ precision for $86 \%$ recall on a real-life dataset containing lots of specific challenges of this application. Indeed, playing children appear in non-standard poses and facial expressions, can be dressed up or have their faces painted etc. In a top-1 sense, our system was able to correctly identify $89.2 \%$ of the faces in close-up. Apart from facial recognition, we discuss and evaluate extending the identification system with person re-identification. To select out the best-looking photos from the identified child photos to fill the album with, we propose an automatic assessment technique that takes into account the aesthetic photo quality as well as the emotions in the photos. Our experiments show that this measure correlates well with a manually labeled general appreciation score.
\end{abstract}

Keywords: Face recognition, Person re-identification, Aesthetics analysis, Emotion classification, Child identification

\section{Introduction}

Many teachers in preschool and elementary school take a large amount of photographs during the school year. These photos form a tangible memory for both child and parent. In many current schools, at the end of the school year, parents receive gigabytes of photographs of all the pictures that were taken that year in class. Mostly, however, parents are only interested in images containing their own child. This forces them to painstakingly go through the enormous amount of images and select only those of interest. To help parents collect photographs of their child without the inconvenience of manually going through thousands of files, we propose an innovative system that automates this whole process. Our system selects pictures that are well-suited candidates for a photo book with a specific child as the main character. For this, we focus on two main aspects: identifying children on a photograph and selecting photographs which are generally perceived as beautiful. 
Both the identification and the aesthetic selection demand a careful procedure to deal with their intrinsic challenges. The identification of children in an environment of school activities, includes identifying children when they are playing, running, dressed up etc. Figure 1 illustrates this with naturally occurring situations where identification is challenging. We propose an approach based on state-of-the-art facial recognition using 8 reference descriptors per identity. Additionally, we developed and employed experiments with a hybrid system that combines facial recognition and state-of-the-art re-ID to handle the highly challenging task of identifying persons of whom the faces are undetectable.
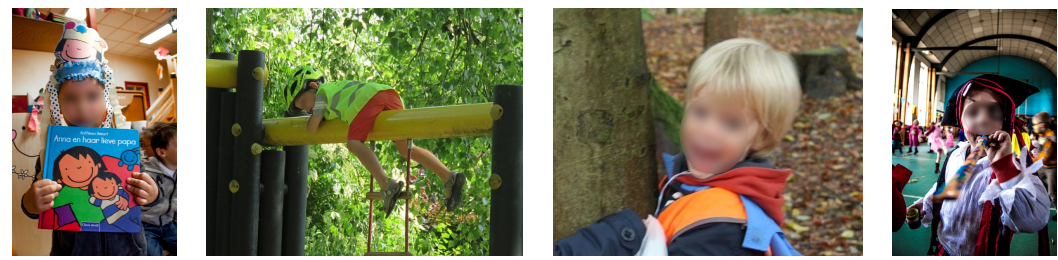

Fig. 1. Examples of challenges in automatic facial recognition for children: dressed up, difficult poses, extreme facial expressions and painted faces.

Due to the underlying subjectivity, selecting the best-looking photographs is another delicate task. We correlated the emotions on photographs and scores on multiple aesthetics criteria with appreciation scores expressed by a panel of human annotators. These correlations suggest a general preference towards positive emotions and compositional aspects like using the rule of thirds. We show that it is - to a certain extent - possible to predict the general appreciation for a photograph by combining emotion classifications with aesthetics criteria scores.

\section{Related Work}

Identifying persons in photo albums has mainly been done in the context of family photo albums 4[10]2. Family photos impose less challenges than pictures taken in a school context. Although the amount of photos might be similar, there are less identities and therefore the amount of photos per identity is higher, increasing the chances for a correct hit. These papers do not take image quality into account and simply focus on the identification aspect, making use of obsolete techniques. Zhao et al. 12, for example, use 2D Hidden Markov Models (HMMs) 2 achieving an Equal Error Rate (EER) of about $40 \%$ precision and recall. Chen et al. 4 propose a block wavelet approach, but only focus on semiautomatic person recognition, i.e. their system suggests a handful identities for a user to select from. Previous literature on image selection based on quality, focuses only on aesthetics criteria [3]. Although important, we are convinced 
that not only the aesthetic quality of the image must be taken into account, but also the expressed emotion of the faces in the photograph. The contribution of our paper is twofold: (i) apply state-of-the-art person identification techniques to the problem of composing a personalized photo album; (ii) use current advances in aesthetics analysis and emotion detection to create an appreciation prediction for selecting the best images for the photo album.

\section{Approach}

Automatically selecting the best photos for an individualized photo album involves two tasks. Firstly, one has to recognize the identities in the photos in order to select those having a specific child as the main character (Section 3.1). From the set of selected photos, in a second step only the most beautiful pictures have to be selected for the album. For this, we propose a photo appreciation scoring approach (Section 3.2), combining both aesthetic and emotional criteria.

\subsection{Identification}

Figure 2 shows a schematic overview of how our proposed system identifies children on a given photo 1 It combines the results of two identification techniques: facial recognition and re-ID. The facial recognition branch consists of three steps. First, a face detector detects the faces that are present in the image. Then the image is categorized into one of four shot types: (i) ambiance photo: a picture without any faces; (ii) group photo: a photo with more than four faces; (iii) close-up: a picture containing at least one face that covers more than $10 \%$ of the total picture area; (iv) non-close-up: a photo that is neither of the previous types. Only for close-up and non-close-up photos, the recognition step is actually performed. We chose to use the Dlib framework 5 because of its state-of-the-art accuracy of $99.38 \%$ on the LFIW dataset. 6] The Dlib face recognition algorithm represents each detected face with a 128-dimensional descriptor vector. By comparing these descriptors with a gallery of reference descriptors of which the identity is known, we estimate the query's identity. The gallery of reference photos is given beforehand and is labeled by, for example, teachers or parents.

Our use case demands the identification of children during everyday activities. This includes identifying children that are playing, running etc. without actually posing in front of the camera. Hence, it is likely that many of the images will not contain the entire face of the child that was photographed. This requires an additional identification approach, apart from facial recognition. Therefore, we added a re-ID branch to our identification system. Person re-identification (re-ID) is the task of re-identifying a person in different images by making use of features from over the entire body, taking into account e.g. the colours of their clothing. In the re-ID branch shown in orange in Figure 2 a person detector first delimits regions where persons are present, i.e. regions containing a head, a body

\footnotetext{
$\overline{1}$ The names are, of course, fictitious.
} 
etc. Next, the detected persons are compared with images of persons whose identities were already estimated. This is done by representing both the identified persons (gallery) and the unidentified persons (query) with a descriptor vector and comparing the unidentified descriptors with the identified descriptors. We trained a ResNet-34 [1]6] network on the CUHK03 [9] dataset using the OpenReID [1] framework which extracts these descriptors. It is important to note that the re-ID comparison is done within the same date, i.e. a given query image will be compared with gallery images that were taken on the same date. That is because the general appearance of a person's body might drastically change every day, mainly due to different clothing.

The facial recognition branch and the re-ID branch are combined to obtain a final estimation of the identities on the image. Once these identities are obtained, the image is added to the re-ID collection of identified photos taken on the respective date. Hence, the re-ID gallery will be gradually extended with potential hits for a given person query.

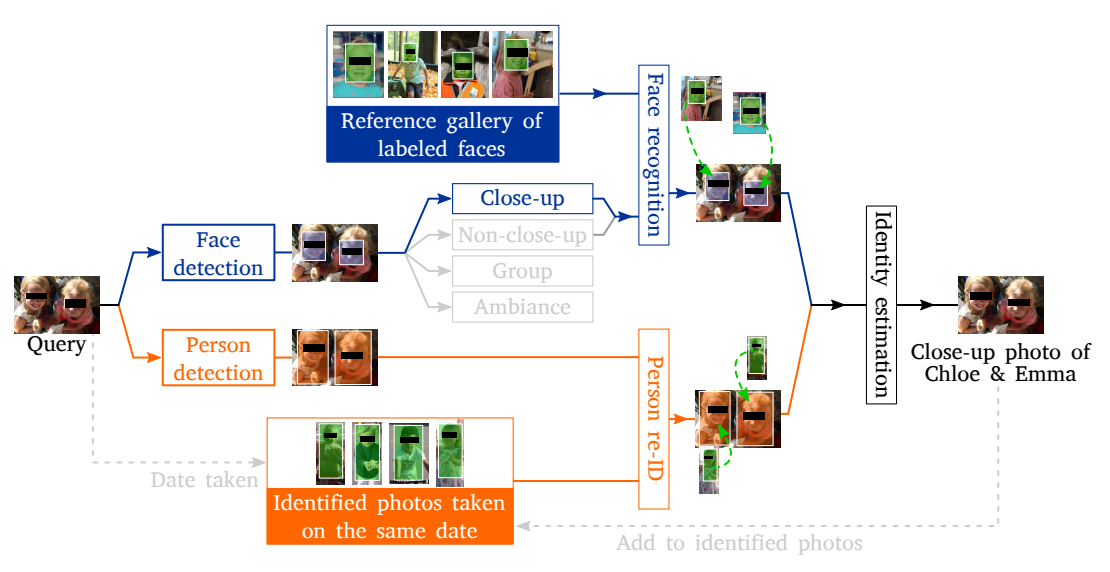

Fig. 2. Overview of the identification part.

\subsection{Estimating Appreciation}

For an individualized child photo album to be satisfactory, it not only should be actually individualized, but it should also contain photographs that are generally perceived as beautiful. Because parents evidently prefer photos in which their children are smiling rather than crying, we combined aesthetics analysis and emotion detection. Figure 3 shows a schematic overview of the appreciation estimation component. The first branch in the diagram contains an emotion classifier. This classifier [1] detects faces in a given photograph and assigns a score to the face for each of seven emotions: happy, surprise, angry, disgust, fear, sad, neutral. The second branch makes use of the Deep Image Aesthetics Analysis 
(DIAA) [7] framework which consists of a Convolutional Neural Network (CNN) that was trained on a dataset with photos rated by professional photographers. Each photo received a rating on multiple criteria (rule of thirds, depth of field etc.) and a general aesthetics score ("Aesthetics" in the diagram). All aesthetics and emotion features are linearly combined to obtain a single appreciation estimation.

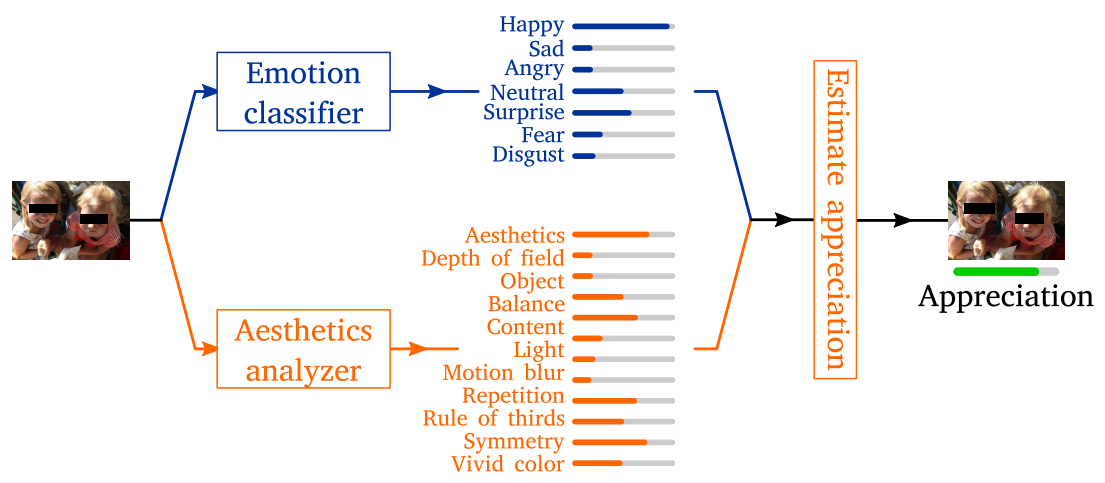

Fig. 3. Overview of the appreciation estimation part.

\section{Results}

For our experiments, we used a dataset of 2086 photos taken by the teacher of a class during the entire school year. It consists of images from children both inside the classroom (during arts and crafts activities, birthdays etc.) and outside the classroom (during a library visit, an ice skating activity etc.). Figure 1 shows some examples of images in the dataset. We annotated the dataset for both the faces and bodies of 33 identities. As can be seen from Figure 4 , there are great differences in the amount of images for each shot type, with specifically a large amount of non-close-up images. To thoroughly evaluate our proposed system, we performed experiments for each specific component of the pipeline. This provides a more detailed analysis than simply evaluating the system as a whole.

\subsection{Face Recognition}

As described in Section 3.1, the facial recognition algorithm yields a descriptor for each detected face. To evaluate the performance of the facial recognition algorithm, we (i) extracted a descriptor for each face in the labeled dataset; (ii) assigned the corresponding identity to each descriptor using the manual annotations; (iii) randomly split up the set of descriptors into a gallery set and a query set such that each identity had $N \geq 1$ descriptors in the gallery which 


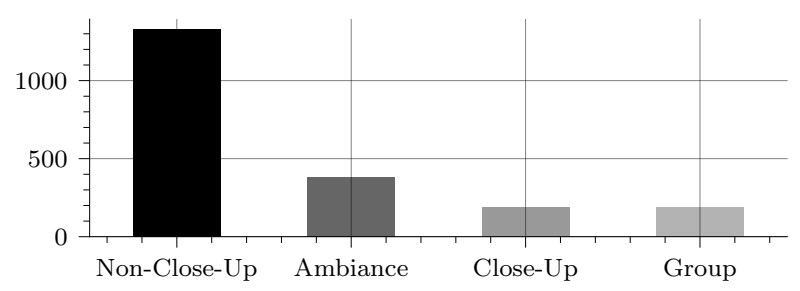

Fig. 4. The distribution of shot types for our dataset.

were used as reference descriptors for that identity; (iv) for each descriptor in the query set, looked for the descriptor in the gallery set to which it had the smallest Euclidean distance (i.e. its nearest neighbour); (v) checked to which extent the labeled identity of the query images matched the identity of their nearest neighbour in the gallery set.

In a first experiment, we examined the influence on the facial recognition accuracy of the amount of reference photos on the one hand, and the averaging of reference descriptors on the other. For the full dataset, we compared the mean Average Precision (mAP) with the amount of gallery descriptors chosen per identity ( $N$ in step (iii) above). Each query descriptor was matched with its nearest neighbour in the gallery. The mAP was calculated for each $N \in$ $\{1,2, \ldots, 15\}$. This experiment was performed for two types of galleries: one where the $N$ descriptors were averaged to always obtain a single descriptor per identity (Fig. 5b) and one where the $N$ descriptors per identity were left untouched (Fig. 5a). The vertical bars in Figure 5 mark the standard deviations for 5 experiments. As we can see, the mAP only increases for the case where the gallery descriptors are averaged. This is mainly because the average of an inlier and an outlier reference descriptor will be less of an outlier. By averaging over multiple reference descriptor, the average will get closer and closer to a reliable representation. When the reference descriptors are not averaged, $N$ has no influence on the accuracy because outliers will be present, no matter the value of $N$. From Fig. 5 , we see that the optimal choice is to average the descriptors with $N=8$, since the mAP hardly increases after $N=8$ with $\mathrm{mAP} \approx 83 \%$.

Our second facial recognition experiment (Figure 6), compares the PrecisionRecall (PR) curves for non-averaged and averaged galleries with $N=8$. We split up the dataset in the shot types described in Section 3.1 to be able to get more detailed insights. Ambiance photos are, of course, not relevant here since they do not contain any faces. It can be seen that the overall performance when using averaged reference descriptors is indeed significantly better with an EER of $86 \%$ precision and $86 \%$ recall for close-up photos. There is, however, a big difference between the shot types. Evidently, the visibility of a face determines the performance of the facial recognition algorithm. In group photos, the faces are a lot smaller and the chances of occlusion are higher compared to close-up photos. 


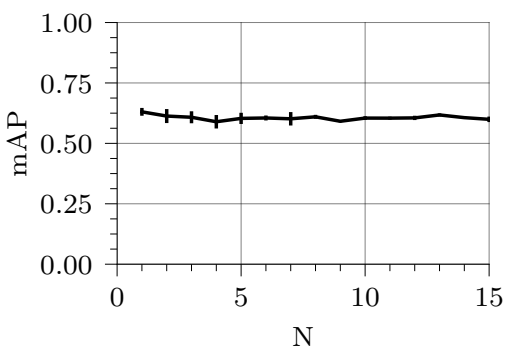

(a) $N$ reference descriptors

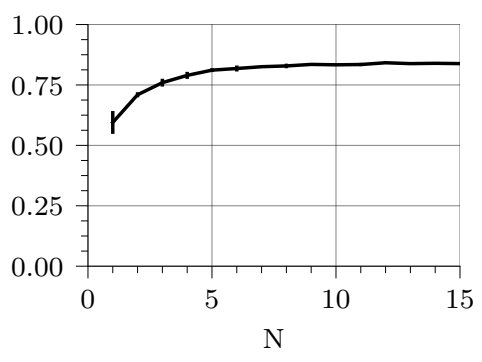

(b) One averaged reference descriptor

Fig. 5. The mAP per gallery size when using facial recognition with (a) all reference descriptors per identity; (b) one averaged reference descriptor.

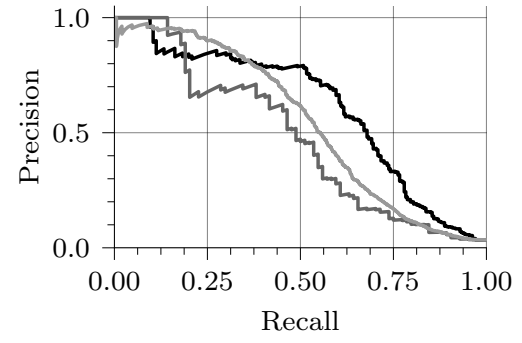

(a) $N$ reference descriptors

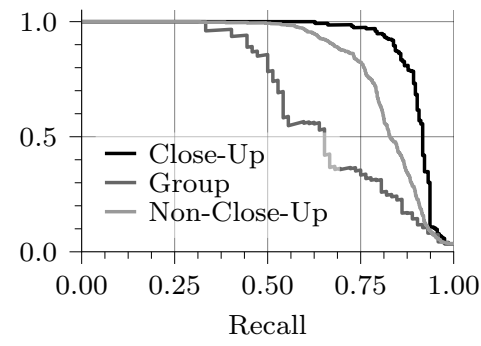

(b) One averaged reference descriptor

Fig. 6. PR curves when using facial recognition $(N=8)$ using (a) all $N$ reference descriptors per identity; (b) a single averaged reference descriptor per identity.

\subsection{Person Re-identification}

In order to be able to identify challenging images where no faces could be detected, we propose to use re-ID (see Fig. 2). The re-ID framework we integrated [11, returns a 128-dimensional descriptor for a given image of a person.

To evaluate the potential of re-ID for our use case, we (i) extracted a descriptor for each person image in the labeled dataset; (ii) assigned the corresponding identity to each descriptor using manual annotations; (iii) added all the descriptors to both the query and the gallery set; (iv) for each descriptor in the query set, looked for the descriptor in the gallery set to which it had the smallest Euclidean distance (i.e. its nearest neighbour), excluding itself; (v) checked to which extent the labeled identity of the query images matched the identity of their nearest neighbour in the gallery set. 


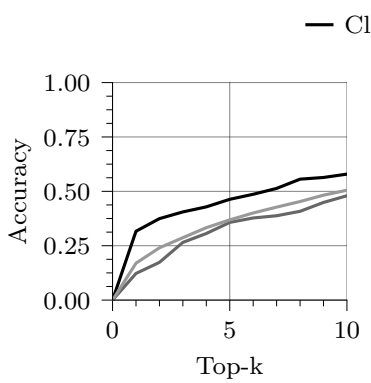

(a) Not taking date into account

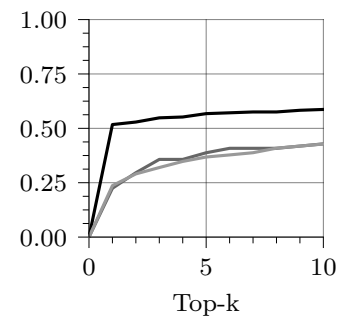

(b) Query and gallery must be of same date

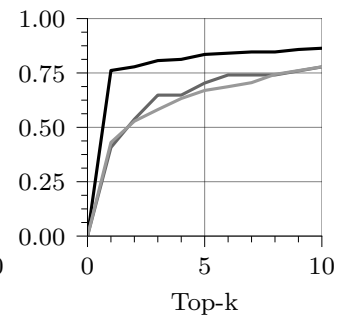

(c) Queries must have match in gallery

Fig. 7. CMC curves when using re-ID (a) not taking the date into account (b) taking the date into account (c) taking the date into account and filtering out queries with no gallery match.

As a first experiment, we investigated how the performance increases when we impose that gallery and query must be of the same date. We created a CMC curve for the case that a query and its matched gallery item can be of different dates (Fig.7a), and one for the case that they must be of the same date (Fig.7b). A CMC curve shows the percentage of queries for which the correct identity is in the top- $k$ nearest gallery descriptors for $k \in\left\{1,2, \ldots, k_{\max }\right\}$. We see that the top-1 accuracy is about $20 \%$ higher for close-ups when we impose gallery and query to be of the same date. It is remarkable, however, that the CMC curve does not seem to converge to $100 \%$ in the same-date case. Indeed, when we plotted this curve for $k_{\max }=$ \#gallery items we saw that it instead converged to a value of about $60 \%$. This means that it was impossible for about $40 \%$ of the queries to match with a correct gallery item of the same date, because there were none. In other words, there is a considerable amount of photos for which the person on the photo was only photographed once that day. Hence, in our use case, reID is intrinsically restricted in accuracy due to its need for same-date images. Therefore, in Figure 7c, we show a similar plot as in Fig. 7b, but now queries for which there were no possible gallery matches were left out. This figure shows that for about $80 \%$ of the (close-up) queries, the nearest neighbour in the gallery is a true match, given two restrictions: (i) a query image can only be matched with a gallery image that was taken on the same day; (ii) each person that is photographed on a certain day must have a corresponding gallery image of that same day.

In a second experiment, we analyzed the complementarity of facial recognition and re-ID. The hypothesis was that we would get a better accuracy by combining re-ID with facial recognition. We applied re-ID wherever the face detector did not detect a face while there was a person present in the image, according to manual annotations. Figure 8a shows the CMC curve of facial recog- 
nition by itself (using $N=8$ averaged gallery descriptors per identity). Figure $8 \mathrm{~b}$ shows the CMC curve of the combination of facial recognition and re-ID. The addition of a state-of-the-art re-ID algorithm did not seem to improve the accuracy of the identification system. It is important to note, however, that there are significant differences between the CUHK03 9 dataset on which the re-ID model was trained, and our own dataset. CUHK03 consists mainly of pedestrians with, more or less, their entire bodies present in the image. Images where a child's body is entirely present, are rather uncommon in our dataset. Future research is needed to examine the possibilities of developing a re-ID model based on datasets that incorporate similar challenges as our own dataset.

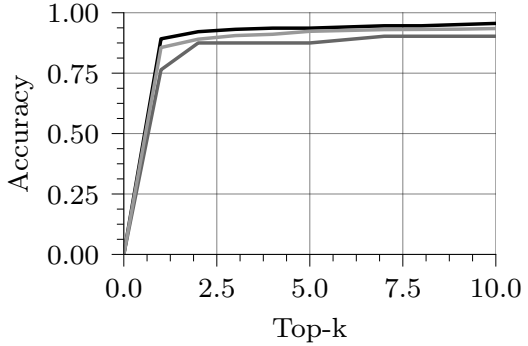

(a) Only facial recognition

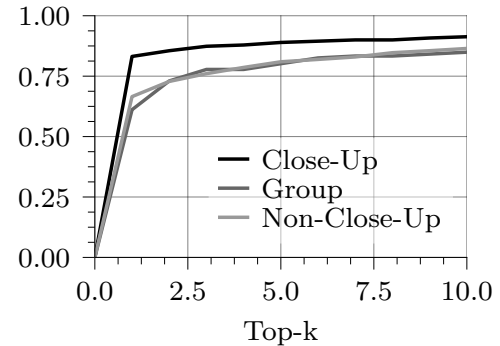

(b) Face recognition and re-ID

Fig. 8. CMC curves for (a) facial recognition $(N=8$, averaged); (b) a combination of facial recognition and re-ID.

\subsection{Estimating Appreciation}

To gather validation data and get an idea about how beautiful a picture is generally perceived, we created a website on which human annotators could label photos as either good or bad. These annotators were selected to be representative for the target audience of parents with children in preschool or elementary school. For each picture, we calculated the average amount of positive votes and used that average as a single appreciation score for the picture. When performing our experiments, each picture was viewed by an average of 9 annotators. As we discussed in Section 3.2. the aesthetics analysis framework returns scores for a set of aesthetics criteria and the emotion detection framework returns - per face - a score for each of seven emotions. For images with multiple faces (and therefore, multiple sets of emotion scores), the emotion scores were averaged by weighing them with the area of the corresponding face detection.

In a first experiment, we calculated the correlations between the features (aesthetics and emotion) and the average appreciation score given by the human annotators. Figure 9 shows the correlations with the largest absolute values, 


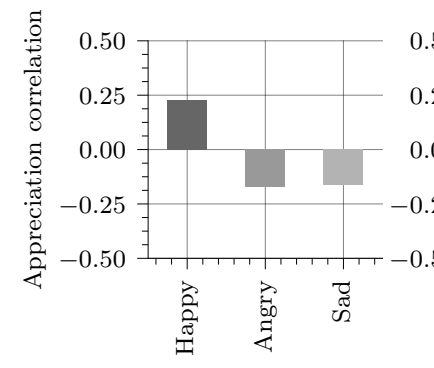

(a) Close-up

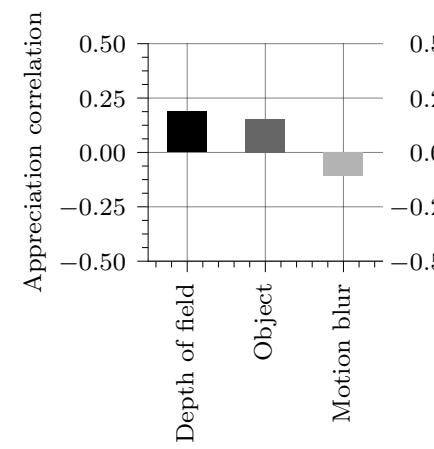

(d) Close-up

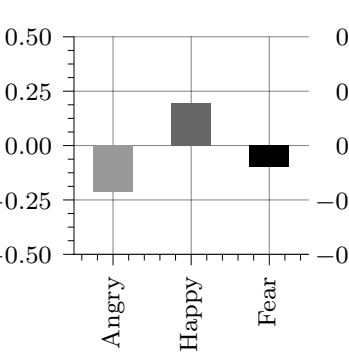

(b) Non-Close-up

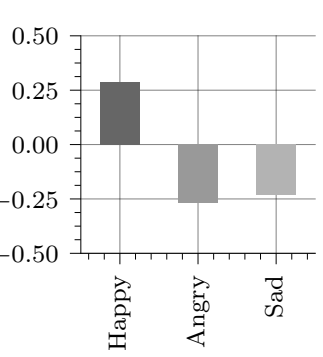

(c) Group

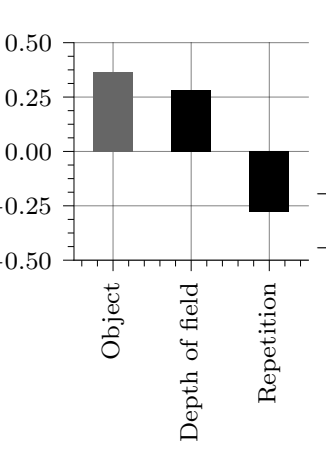

(e) Non-Close-up

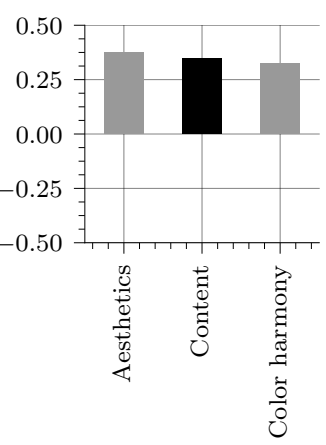

(f) Group

Fig. 9. Highest correlations (in absolute value) between (a) (c) annotators appreciation and emotion; (d) (f) annotators appreciation and aesthetic features.

for emotions and aesthetics criteria respectively. Bars that are oriented upward, correlate positively with the average score given by annotators, while bars that are oriented downward, correlate negatively. On Figs. 9a 9c, we see that positive emotions (happy) tend to correlate positively with the opinion of the human annotators while negative emotions (angry, sad) tend to correlate negatively. This means that, in general, the annotators preferred pictures with positive emotions over pictures with negative emotions, as could be expected beforehand. Figures $9 \mathrm{~d}[9 \mathrm{f}]$ show that the annotators mostly appreciated a shallow depth of field and a clear focus on a single object (person) in close-up and non-close-up photos. For group photos, the general aesthetics score seems to be an important factor for the appreciation.

As explained before, we propose to linearly combine these different scores into one appreciation score. By employing a ridge regression, we derive that linear combination of the aesthetics scores and the emotion scores. We trained 10 linear regression models for each shot type and selected those with the highest R2-score. Next, these models were evaluated on a test set. Figure 10 shows the highest 
correlations (for the test set) between a set of features - aesthetics, emotion and the trained linear combination of these - and the average appreciation from the annotators. The correlation between the trained linear feature combination and the average annotator appreciation is indicated with "Prediction" in Fig. 10. A clear advantage is found for non-close-up images where the prediction is the most correlated feature in the test set. For close-up and group images, however, the prediction correlates for 0.04 and -0.27 , respectively; which is less than the 6 most correlated features shown in Figs. 10a and 10c It is possible, however, that due to the small amount of close-up and group images present in the dataset (see also Fig. 4), the test sets for these shot types were not representative. This can also be seen when comparing Figs. 9 and 10 . For the non-close-up test set in Fig. 10b, the correlations are similar to those in Figs. $9 \mathrm{~b}$ and $9 \mathrm{e}$. For the correlations of the features in the close-up and group test set, however, this is less the case.

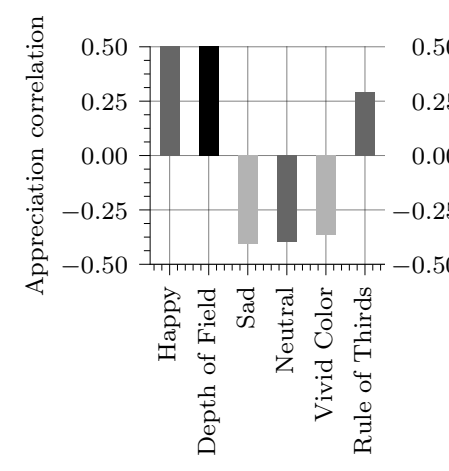

(a) Close-up

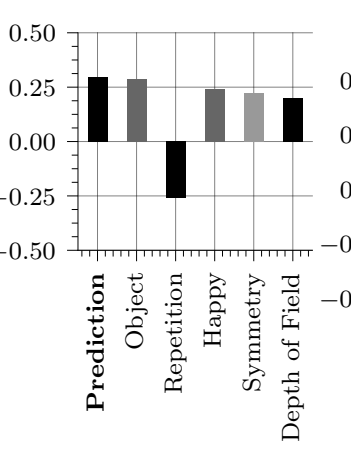

(b) Non-Close-up

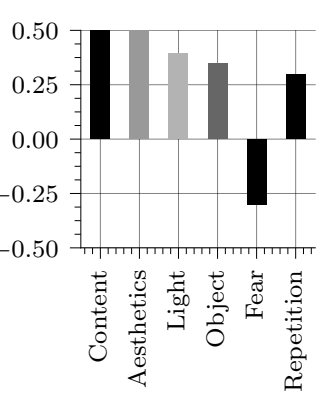

(c) Group

Fig. 10. Correlations with human annotators appreciation and linear regression prediction test set.

\section{Conclusion}

In this paper, we proposed a system to automate the individualized selection of child photos taken during a school year. Our approach consisted of two parts: correctly identifying children on photographs and assigning an appreciation score to each image.

We showed that facial recognition is very suitable for our use case, especially when taking the average of about 8 reference descriptors to obtain a single reference face descriptor per identity. We obtained $86 \%$ precision for $86 \%$ recall. We also presented that the facial recognition technique by itself is able to achieve a top-1 accuracy of $89.2 \%$. Furthermore, we evaluated the accuracy gain of adding 
re-ID to the identification system. This, however, resulted in a decrease in accuracy.

We proposed a method of estimating the appreciation generally expressed by our target audience with respect to given images. For non-close-up photos, the prediction model combined the emotion and aesthetics features in a way to obtain a better correlation with the scores given by the annotators.

Future research needs to point out whether a prediction model can be obtained that works for all shot types by using (i) a more representative appreciation questionnaire with, for example, at least 30 annotations per image; (ii) other aesthetics analysis or emotion detection frameworks, eventually more aimed at child photos; (iii) a complexer prediction model (e.g. a neural network).

\section{References}

1. Arriaga, O., Valdenegro-Toro, M., Plöger, P.: Real-time convolutional neural networks for emotion and gender classification. arXiv preprint arXiv:1710.07557 (2017)

2. Cardinaux, F., Sanderson, C., Bengio, S.: Face verification using adapted generative models. In: Automatic Face and Gesture Recognition, 2004. Proceedings. Sixth IEEE International Conference on. pp. 825-830. IEEE (2004)

3. Ceroni, A., Solachidis, V., Niederée, C., Papadopoulou, O., Kanhabua, N., Mezaris, V.: To keep or not to keep: An expectation-oriented photo selection method for personal photo collections. In: Proceedings of the 5th ACM on International Conference on Multimedia Retrieval. pp. 187-194. ACM (2015)

4. Chen, L., Hu, B., Zhang, L., Li, M., Zhang, H.: Face annotation for family photo album management. International Journal of Image and Graphics 3(01), 81-94 (2003)

5. King, D.E.: Dlib-ml: A machine learning toolkit. Journal of Machine Learning Research 10(Jul), 1755-1758 (2009)

6. King, D.E.: High quality face recognition with deep metric learning (2017), http: //blog.dlib.net/2017/02/high-quality-face-recognition-with-deep.html

7. Kong, S., Shen, X., Lin, Z., Mech, R., Fowlkes, C.: Photo aesthetics ranking network with attributes and content adaptation. In: ECCV (2016)

8. Li, C., Loui, A.C., Chen, T.: Towards aesthetics: A photo quality assessment and photo selection system. In: Proceedings of the 18th ACM international conference on Multimedia. pp. 827-830. ACM (2010)

9. Li, W., Zhao, R., Xiao, T., Wang, X.: Deepreid: Deep filter pairing neural network for person re-identification. In: CVPR (2014)

10. O'Hare, N., Smeaton, A.F.: Context-aware person identification in personal photo collections. IEEE Transactions on Multimedia 11(2), 220-228 (2009)

11. Xiao, T.: open-reid (2017), https://github.com/Cysu/open-reid

12. Zhao, M., Teo, Y.W., Liu, S., Chua, T.S., Jain, R.: Automatic person annotation of family photo album. In: International Conference on Image and Video Retrieval. pp. 163-172. Springer (2006) 